\title{
Qué es el Instituto de Investigaciones en Ciencias Financieras y Contables
}

\author{
Dr. Raúl Arrarte Mera ${ }^{1}$ \\ Docente Asociado de la Facultad de Ciencias Contables
}

\section{RESUMEN}

El objetivo del presente articulo es hacer de conocimiento público de las obligaciones y responsabilidades que desarrolla nuestro Instituto de Investigaciones por encargo del Consejo Superior de Investigaciones, dependiente del Vicerrectorado de Investigación de la UNMSM.

Se desarrolló un análisis de la Resolución Rectoral No.05680-R-08, vigente a partir del 04 de diciembre de 2008, de naturaleza descriptivo-explicativo y se utilizó los métodos inductivo y de análisis documental.

Si deseamos obtener una Acreditación Internacional como Facultad de Ciencias Contables, es obligación indispensable de sus Docentes a Dedicación Exclusiva y Tiempo Completo, dedicarse con pasión, al fomento de la investigación aplicativa y multidisciplinaria.

Pese a sus escasos recursos, el Vicerrectorado de Investigación estimula la dedicación del docente y del estudiante a las actividades de Investigación+Desarrollo+Innovación, no obstante, muchas de estas investigaciones, en caso particular de la Facultad de Ciencias Contables, no son aplicativas para los tiempos que nos toca vivir en una sociedad globalizada.

Palabras clave: Investigación universitaria, Investigación y desarrollo, Investigación e innovación, Investigación multidisciplinaria.

1 Actual Director del Instituto de Investigaciones de la Facultad de Ciencias Contables. 


\section{INTRODUCCIÓN}

La Facultad de Ciencias Contables, a través del Instituto de Investigación de Ciencias Financieras y Contables, por intermedio del Vicerrectorado de Investigación, promueve los siguientes programas:

\section{A NIVEL DE DOCENTES}

\section{Estudios de Investigación en la Facultad.}

Se desarrollan en tres modalidades basadas en la fuente de financiamiento y la subvención otorgada por realizar dicha actividad:

A. Estudios Con Asignación a la Investigación y Con Incentivos al Investigador (CON-CON). El número de estudios de investigación por Facultad a ser aprobado es fijado anticipadamente por el CSI e informado a las respectivas Facultades. Cantidad que será fijada en base a los siguientes aspectos:

- Disponibilidad de recursos.

- Número de estudios de investigación ejecutados en el ańo.

- Número de docentes nombrados a dedicación exclusiva y tiempo completo.

- Número de investigadores con grado de Magíster y de Doctor.

- Número de publicaciones científicas especializadas en revistas indexadas que se encuentran registradas.

- Número de Tesis de Pregrado y Postgrado defendidas.

- Número de estudios o proyectos de investigación con financiamiento externo obtenidos.

- Grado de cumplimiento de las actividades de investigación.

B. Estudios Sin Asignación a la Investigación y Con Incentivo al Investigador (SIN-CON), Los Estudios SIN-CON son aquellos proyectos de investigación financiadas por otras instituciones nacionales o extranjeras, en los cuales participan investigadores de la universidad; estos estudios no cuentan con Asignación a la Investigación pero sí con Incentivo al Investigador. Estudios que deben acreditar recursos anuales superiores al monto señalado oportunamente por el CSI, los cuales ingresan a la Facultad respectiva en dinero, bienes o servicios. Las propuestas de Estudios SIN-CON deben presentarse con una copia del convenio, acuerdo o contrato de investigación con la institución financiadota.

C. Estudios Sin Asignación a la Investigación y Sin Incentivo al Investigador (SIN-SIN), Son financiados por los Profesores Investigadores, las Facultades o por otras instituciones. Los investigadores que participen en este tipo de estudios no son beneficiarios del Incentivo al Investigador por limitaciones presupuestales de la universidad pero su esfuerzo científico será reconocido y registrado por CSI. Además son partícipes de los beneficios indicados en el Artículo $5^{\circ}$.

\section{Programas de Proyectos Multidisciplinarios}

Tienen el propósito de contribuir al desarrollo de capacidades en la formulación, diseño y elaboración de proyectos que permitan resolver problemas de carácter estratégico en el país. Las áreas de investigación son:

A. Ciencias Naturales y de la Vida.

B. Ciencias Tecnológicas y,

C. Ciencias Sociales-Humanas.

\section{Mérito científico}

Premio otorgado a los investigadores más destacados de las diversas facultades de la 
universidad en mérito a sus publicaciones registradas en el CSI (artículos en revistas, libros y capítulos de libros), grados y títulos del investigador, participación en proyectos de investigación con financiamiento externo y las distinciones académicas que haya recibido.

\section{Programas de Reconocimiento a las actividades de investigación de los profesores de la UNMSM}

A. Promoción de Asesorías de Tesis de Pregrado en la UNMSM. Otorgado a los Docentes Asesores de tesis de pre y postgrado (Magíster y Doctor).

B. Promoción de las Publicaciones Científicas. Otorgado en los siguientes casos:

- Artículos científicos originales e inéditos registrados en base de datos ISI.
- Artículos originales e inéditos publicados en revistas científicas con ISSN.

- Libro especializado con ISBN derivado de un trabajo de investigación, publicado por editorial de prestigio.

- Capítulo de libro especializado con ISBN derivado de un trabajo de investigación, publicado por editorial de prestigio.

C. Promoción para la obtención del grado de Maestro y Doctor de los docentes de la UNMSM. Otorgado a los Docentes que hayan obtenido el grado académico de Maestro o Doctor.

Veamos a continuación algunos cuadros estadísticos de los Docentes de la Facultad de Ciencias Contables y su relación con la Investigación:

PROFESORES INVESTIGADORES CON DERECHO A PRESENTAR PROYECTOS AL 31.12.2008

\begin{tabular}{|c|l|c|c|}
\hline No. NOMBRES Y APELLIDOS & PUNTAJE TOTAL & ÚltimOS 5 AÑOS \\
\hline 1 & Gutiérrez Huby, Ana María & 20.00 & 12.50 \\
\hline 2 & Arrarte Mera, Raúl Alberto & 29.75 & 10.25 \\
\hline 3 & Choy Zevallos, Elsa Esther & 18.00 & 8.50 \\
\hline 4 & Wong Torres, Zelma & 21.25 & 7.25 \\
\hline 5 & Palomino Silva, Julio & 13.25 & 7.25 \\
\hline 6 & Gomero Gonzáles, Nicko & 18.50 & 7.00 \\
\hline 7 & Flores Konja, Adrián Alejandro & 16.00 & 6.00 \\
\hline 8 & Egúsquiza Pereda, Carlos Alfonso & 28.25 & 5.75 \\
\hline 9 & Bortesi Longhi, Luis & 14.50 & 5.25 \\
\hline 10 & Polar Falcón, Ernesto & 99.75 & 5.00 \\
\hline
\end{tabular}

FUENTE: Consejo Superior de Investigaciones

COMENTARIO: Los Investigadores que figuran en esta lista para ser Responsables de Proyectos necesitan como mínimo 12 puntos del puntaje total y cinco puntos en los últimos cinco años.

RELACIÓN DE PROYECTOS DE INVESTIGACIÓN CON - CON RESPONSABLES DE ESTUDIOS REALIZADOS EN LOS 6 ÚLTIMOS AÑOS

\begin{tabular}{|l|c|c|c|c|c|c|}
\hline \multirow{2}{*}{\multicolumn{1}{c|}{ Nombres Y APELLIDOS }} & \multicolumn{7}{c|}{ RESPONSABLES DE LOS PROYECTOS } \\
\cline { 2 - 7 } & 2009 & 2008 & 2007 & 2006 & 2005 & 2004 \\
\hline Angulo Silva, Luis Ángel & 0 & 0 & 0 & 0 & 0 & 0 \\
\hline Arrarte Mera, Raúl Alberto & Sí & No & Sí & No & No & Sí \\
\hline Ataupillco Vera, Víctor Dante & 0 & 0 & 0 & 0 & 0 & 0 \\
\hline Barrón Araoz, Ampelio, Ricardo & 0 & 0 & 0 & 0 & 0 & 0 \\
\hline Bazán Castillo, Santiago & 0 & 0 & 0 & 0 & 0 & 0 \\
\hline
\end{tabular}




\begin{tabular}{|c|c|c|c|c|c|c|}
\hline Bortesi Longhi, Luís & Sí & Sí & Sí & Sí & No & No \\
\hline Choy Zevallos, Elsa ${ }^{*}$ ) & No & Sí & No & No & No & No \\
\hline Díaz Inchicaqui, Miguel $\left(^{*}\right)$ & No & No & No & No & Sí & No \\
\hline Egúsquiza Pereda, Carlos Alfonso & Sí & No & Sí & Sí & Sí & Sí \\
\hline Flores Konja, Adrián $\left(^{*}\right)$ & Sí & Sí & Sí & No & Sí & Sí \\
\hline Flores Konja, Julio Vicente & 0 & 0 & 0 & 0 & 0 & 0 \\
\hline Gavelán Izaguirre, Jorge & 0 & 0 & 0 & 0 & 0 & 0 \\
\hline Gomero Gonzáles, Nicko & Sí & Sí & Sí & Sí & No & No \\
\hline Gutiérrez Carillo, Jesús & 0 & 0 & 0 & 0 & 0 & 0 \\
\hline Gutiérrez Huby, Ana María & No & Sí & No & No & No & No \\
\hline Herrera García, Beatriz & No & No & No & Sí & Sí & Sí \\
\hline Javier Melgarejo, Leoncio & No & No & No & No & Sí & No \\
\hline León Fernández, Bonifacio & 0 & 0 & 0 & 0 & 0 & 0 \\
\hline Miñano Lecaros, Juan Guillermo $\left(^{*}\right)$ & No & No & No & No & Sí & No \\
\hline Montes Farro, Eduardo Alberto & 0 & 0 & 0 & 0 & 0 & 0 \\
\hline Polar Falcón, Ernesto Augusto & Sí & Sí & Sí & No & No & Sí \\
\hline Ramón Ruffner, Jerí Gloria & No & No & No & No & No & Sí \\
\hline Rodríguez Cairo, Vlaldimir & No & No & No & No & Sí & No \\
\hline Rozas Flores, Alan Errol & No & No & No & No & Sí & No \\
\hline Salcedo Guzmán, Luisa Elena & 0 & 0 & 0 & 0 & 0 & 0 \\
\hline Trujillo Meza, Julio & 0 & 0 & 0 & 0 & 0 & 0 \\
\hline Talla Ramos, Sabino & 0 & 0 & 0 & 0 & 0 & 0 \\
\hline Ugarte Silva, Saturnino Alfonso & No & Sí & Sí & Sí & No & No \\
\hline Vilchez Olivares, Percy & 0 & 0 & 0 & 0 & 0 & 0 \\
\hline Wong Torres, Zelma & Sí & No & No & Sí & Sí & Sí \\
\hline
\end{tabular}

${ }^{*}$ ) Docente a Tiempo Parcial.

Fuente. Archivos del Instituto de Investigaciones de Ciencias Financieras y Contables.

PERSONAL A DEDICACIÓN EXCLUSIVA Y TIEMPO COMPLETO OBLIGADO A DESARROLLAR ACTIVIDADES DE INVESTIGACIÓN

\begin{tabular}{|c|l|c|c|}
\hline N. $^{\circ}$ & \multicolumn{1}{|c|}{ NomBRES Y APELLIDOS } & CLASE & INVESTIGADOR VIGENTE \\
\hline 1 & Angulo Silva, Luis Ángel & T. C. & No \\
\hline 2 & Arrarte Mera, Raúl Alberto & T. C. & Sí \\
\hline 3 & Ataupillco Vera, Víctor Dante & T. C. & No \\
\hline 4 & Barrón Araoz, Ampelio, Ricardo & T. C. & No \\
\hline 5 & Bazán Castillo, Santiago & T. C. & Sí \\
\hline 6 & Bortesi Longhi, Luís & T. C. & Sí \\
\hline 7 & Egúsquiza Pereda, Carlos Alfonso & T. C. & No \\
\hline 8 & Flores Konja, Julio Vicente & T. C. & Sí \\
\hline 9 & Gavelán Izaguirre, Jorge & T. C. & Sí \\
\hline 10 & Gomero Gonzáles, Nicko & T. C. & Sí \\
\hline 11 & Gutiérrez Carillo, Jesús & T. C. & No \\
\hline 12 & Gutiérrez Huby, Ana María & T. C. & No \\
\hline 13 & Herrera García, Beatriz & D. E. & No \\
\hline 14 & Javier Melgarejo, Leoncio & T. C. & T. \\
\hline 15 & León Fernández, Bonifacio & & . \\
\hline
\end{tabular}




\begin{tabular}{|l|l|l|l|}
\hline 16 & Montes Farro, Eduardo Alberto & T. C. & No \\
\hline 17 & Polar Falcón, Ernesto Augusto & T. C. & Sí \\
\hline 18 & Ramón Ruffner de la Vega, Jerí Gloria & T. C. & No \\
\hline 19 & Rodríguez Cairo, Vlaldimir & T. C. & No \\
\hline 20 & Rozas Flores, Alan Errol & T. C. & No \\
\hline 21 & Salcedo Guzmán, Luisa Elena & T. C. & No \\
\hline 22 & Talla Ramos, Sabino & T. C. & No \\
\hline 23 & Trujillo Meza, Julio & T. C. & No \\
\hline 24 & Ugarte Silva, Saturnino Alfonso & T. C. & No \\
\hline 25 & Vílchez Olivares, Percy & T. C. & Sí \\
\hline 26 & Wong Torres, Zelma & T. C. & \\
\hline
\end{tabular}

Fuente: Archivos de Dirección de Coordinación Académica e IICFC.

\section{PERSONAL A DEDICACIÓN EXCLUSIVA Y TIEMPO COMPLETO CON GRADO DE MAGÍSTER Y DOCTOR}

\begin{tabular}{|c|c|c|c|c|}
\hline No. & NOMBRES Y APELLIDOS & MAGISTER & DOCTOR & INVESTIGADOR VIGENTE \\
\hline 1 & Angulo Sílva, Luis Ángel & 0 & 0 & 0 \\
\hline 2 & Arrarte Mera, Raúl Alberto & Sí & Sí & Sí \\
\hline 3 & Ataupillco Vera, Víctor Dante & Sí & 0 & 0 \\
\hline 4 & Barrón Araoz, Ampelio, Ricardo & Sí & Sí & 0 \\
\hline 5 & Bazán Castillo, Santiago & 0 & 0 & 0 \\
\hline 6 & BorteSí Longhi, Luís & Sí & Sí & Sí \\
\hline 7 & Egúsquiza Pereda, Carlos Alfonso & 0 & Sí & Sí \\
\hline 8 & Flores Konja, Adrián (*) & Sí & Sí & Sí \\
\hline 8 & Flores Konja, Julio Vicente & 0 & Sí & 0 \\
\hline 9 & Gavelán Izaguirre, Jorge & 0 & 0 & 0 \\
\hline 10 & Gomero Gonzáles, Nicko & Sí & Sí & Sí \\
\hline 11 & Granda Carazas, Eloy $\left(^{*}\right)$ & Sí & Sí & No \\
\hline 12 & Gutiérrez Carillo, Jesús & 0 & 0 & 0 \\
\hline 13 & Gutiérrez Huby, Ana María & Sí & 0 & Sí \\
\hline 14 & Herrera García, Beatriz $\left(^{* *}\right)$ & 0 & Sí & 0 \\
\hline 15 & Javier Melgarejo, Leoncio & 0 & 0 & 0 \\
\hline 16 & León Fernández, Bonifacio & 0 & 0 & 0 \\
\hline 17 & Montes Farro, Eduardo Alberto & 0 & 0 & 0 \\
\hline 18 & Polar Falcón, Ernesto Augusto & 0 & Sí & Sí \\
\hline 19 & Ramón Ruffner de la Vega, Jerí Gloria & Sí & Sí & 0 \\
\hline 20 & Rodríguez Cairo, Vlaldimir & Sí & 0 & 0 \\
\hline 21 & Rozas Flores, Alan Errol & 0 & 0 & 0 \\
\hline 22 & Salcedo Guzmán, Luisa Elena & 0 & 0 & 0 \\
\hline 23 & Talla Ramos, Sabino & Sí & Sí & No \\
\hline 24 & Trujillo Meza, Julio & 0 & 0 & 0 \\
\hline 25 & Ugarte Sílva, Saturnino Alfonso & 0 & Sí & 0 \\
\hline 26 & Vílchez Olivares, Percy & Sí & 0 & 0 \\
\hline 27 & Wong Torres, Zelma & Sí & 0 & Sí \\
\hline
\end{tabular}

(*) Docente a tiempo parcial.

$\left(^{* *}\right)$ Docente a dedicación exclusíva.

Fuente: Archivos de Coordinación Académica e IICFC.

COMENTARIO: Nuestra Facultad cuenta con 13 Doctores. Sólo 8 Doctores Investigadores son Vigentes. El Vicerrectorado de Investigación tiene asignado al Instituto de Investigaciones de Ciencias Financieras y Contables, 7 Cupos Con-Con, totalmente financiados

\section{QUIPURAMAYOC}




\section{INVESTIGACIÓN A NIVEL DE ESTUDIANTES}

Los Programas que promueve el Vicerrectorado de Investigación (VRI), a nivel de la Universidad, son los siguientes:

\section{Fondo de Promoción de Trabajo de Tesis de Pregrado}

En adelante denominaremos Fondo, tiene como objetivo fomentar la investigación, a través de tesis, en los estudiantes de Pregrado, así como promover el trabajo conjunto de creación intelectual del profesor y del estudiante.

El Fondo está constituido conjuntamente por el aporte anual del VRI de una Unidad de Financiamiento de Tesis (UFT), y el aporte de la Facultad correspondiente a dos UFT. La UFT es fijada anualmente por el CSI. El monto máximo asignado para cada propuesta de Tesis es de S/.2,000 nuevos soles y está destinado únicamente para financiar gastos operativos de la Tesis.

El CSI convoca anualmente a través de la Unidad de Investigación de cada Facultad, al concurso de méritos para el financiamiento de trabajos de tesis por el Fondo. Los proyectos de tesis que ingresan al concurso de mérito deben estar registrados en la Escuela Académico-Profesional correspondiente.

Cada Facultad debe conformar una Comisión Técnica de Evaluación de Proyectos de Tesis para su financiamiento por el Fondo, en adelante denominada Comisión Técnica, la que es presidida por el Director de Investigaciones e integrada por los Directores de las Escuelas Académico-Profesionales cuyos estudiantes postulan al concurso y el Director Académico de la Facultad.

La Comisión Técnica evalúa los proyectos de tesis presentados al concurso de méritos, considerando los siguientes términos de referencia:
- Generación de conocimiento nuevo.

- Pertinencia y claridad de la hipótesis.

- Objetivos concretos claramente definidos y en concordancia con la hipótesis y

- Metodología coherente con los objetivos planteados.

El acceso al financiamiento por el Fondo será por estricto orden de méritos.

El Director de Investigación eleva al Consejo de Facultad el informe de la Comisión Técnica para su ratificación mediante Resolución de Decanato, la que es elevada al VRI para la emisión de la Resolución Rectoral.

El monto proporcionado por el Fondo es asignado al Asesor de Tesis luego de la emisión luego de la emisión de la Resolución Rectoral correspondiente.

Es de responsabilidad del Asesor de Tesis y del Tesista la presentación de los informes económicos y técnicos a la Unidad de Investigación de su Facultad en las fechas señaladas por el CSI y de acuerdo a las directivas correspondientes.

El informe técnico corresponde a una de las siguientes opciones:

- Un ejemplar de tesis sustentada.

- Un borrador de tesis si ya inició el trámite de la sustentación.

- Una publicación de autoría compartida entre el asesorado y el asesor en unas revista de investigación de la especialidad.

En el caso de que el trabajo realizado no amerite publicación, el asesor de tesis entregará un informe técnico que contará con el aval del Director de la Unidad de Investigación.

\section{Grupos Estudiantiles dedicados a la} Investigación, Innovación y Transferencia Son asociaciones estudiantiles sin fines de lucro, creadas en las Facultades y relacionadas a una o más disciplinas del conocimiento, 
vinculadas a uno o más Institutos de Investigación. El Docente Asesor de estos grupos recibe una asignación económica para financiar sus actividades.

\section{VENTAJAS DE SER INVESTIGADOR EN LA FACULTAD DE CIENCIAS CONTABLES}

Los estudios y las publicaciones científicas aprobados y registrados, son considerados por los investigadores para su carga lectiva, ascensos y promociones en la Universidad, así como justificación para becas, viajes de trabajo y licencias, según sea el caso. Asimismo, sirven como justificativo para el acceso a laboratorios y uso de equipos especializados. Aquellas actividades que no se encuentran registradas no serán reconocidas como actividades oficiales de la Universidad (Art. 50).

Los docentes nombrados de la Universidad, independientemente de la categoría y clase, así como los Profesores Eméritos y Jefes de Práctica nombrados, pueden participar en actividades de investigación y recibir el Incentivo al Investigador. A su vez, otros investigadores pueden colaborar en la ejecución de la investigación

La participación de los investigadores en las Actividades de Investigación se da en los siguientes niveles:

- Responsable.

- Miembro.

- Colaborador.

Los Responsables de Estudios CONCON requieren tener publicaciones científicas registradas. El puntaje mínimo requerido para ser considerado Responsable será señalado oportunamente por el CSI.

Los Responsables de estudios SINCON y SIN-SIN no requieren puntaje en publicaciones. (Art. 43).
Los Miembros de estudios CON-CON podrán ser de Tipo A y Tipo B.

Tipo A: Son los Investigadores que tienen publicaciones científicas registradas. El puntaje mínimo por publicaciones científicas para ser considerado Miembro A será señalado oportunamente por el CSI. El número de miembros Tipo A está limitado por las necesidades técnicas del estudio.

Tipo B: Son Investigadores con puntajes menores a los exigidos para ser miembro tipo A. El puntaje mínimo para ser considerado miembro tipo $\mathrm{B}$ y el número máximo de miembros Tipo $\mathrm{B}$ por estudio, serán señalados oportunamente por el CSI.

Los miembros de Estudios SIN-CON requieren tener publicaciones científicas registradas. El puntaje por publicaciones y el número de Miembros en estos estudios será señalado oportunamente por el CSI.

Los miembros de Estudios SIN-SIN no requieren tener publicaciones científicas registradas. En el registro indicado; el número de Miembros está limitado por las necesidades técnicas del estudio.

Los Colaboradores de los estudios CON-CON, SIN-CON y SIN-SIN no requieren tener publicaciones científicas registradas y pueden ser:

- Docentes de la Universidad, en calidad de nombrados o contratados e indistintamente de su categoría y clases.

- Profesores Eméritos.

- Jefes de Práctica.

- Investigadores de otras universidades e instituciones ajenas a la Universidad de San Marcos.

- Estudiantes de Pre y Post Grado de la Universidad.

El Responsable es aquel investigador que lidera una actividad de investigación 
y se responsabiliza técnica y administrativamente por su ejecución.

El Miembro y el Colaborador son aquellos investigadores que apoyan al Responsable con sus conocimientos y actividad calificada en la planificación, ejecución y análisis de resultados de la actividad de investigación.

\section{CONCLUSIÓN}

La Carrera Profesional del Contador Público es una especialidad íntimamente ligada al devenir de la macro y microeconomía. El día a día es muy intenso y deja poco espacio al Docente para dedicarse, como desearía, a la investigación aplicada a tiempo completo. Aún, desde el punto de vista económico, el incentivo resulta insuficiente comparado con la asesoría privada que puede cobrar, por ese mismo tiempo en el mercado. Fenómeno que se repite en los estudiantes, que como perciben ingresos medios superiores, prefieren pagar un Curso de Titulación, a optar por una Tesis de Investigación financiado por el VRI, porque resulta más larga más allá de los cinco meses que ofrece la primera opción.
Pese a sus escasos recursos, el Vicerrectorado de Investigación estimula la dedicación del docente y del estudiante a las actividades de Investigación+Desarrollo+Innovación, no obstante, muchas de estas investigaciones, en el caso particular de la Facultad de Ciencias Contables, no son aplicativas para los tiempos que nos toca vivir en una sociedad globalizada.

Sin embargo, en el mediano plazo, abrigamos la esperanza de tener en algún momento, un Banco de Proyectos a ser financiados por la Empresa Privada o por las Regiones. Alternativa viable que debe incrementar la cooperación entre Universidad-Empresa y Estado.

\section{REFERENCIAS}

Instituto de Investigaciones de Ciencias $\mathrm{Fi}$ nancieras y Contables. Archivos 20042009 .

Universidad Nacional Mayor de San Marcos. Resolución Rectoral N. ${ }^{\circ}$ 05680-R-08.

Universidad Nacional Mayor de San Marcos. Vicerrectorado de Investigación, Calendario 2009. 